Real Analysis Exchange

Vol. 24(2), 1998/9, pp. 621-633

Ryszard Jerzy Pawlak, Faculty of Mathematics, Łódź University, Banacha 22, 90-238 Łódź, Poland. e-mail: RPAWLAK@imul.uni.lodz.pl

\title{
ON IDEALS OF EXTENSIONS OF RINGS OF CONTINUOUS FUNCTIONS
}

\begin{abstract}
In this paper we consider the properties of ideals of some rings of almost continuous functions, being extensions of rings of continuous functions.
\end{abstract}

\section{Introduction}

Although the properties of rings of real continuous functions have been studied for a long time (cf. the results of [7]), many interesting articles concerning these problems are still appearing, among others, in reference to the theory of ideals of these rings (e.g. [1],[8]). Investigations of rings wider than rings of continuous functions are also carried out (e.g.[18]).

In 1959 J. Stallings introduced (in order to generalize the Brouwer fixed point theorem) the notion of almost continuity.

A function $f: X \rightarrow Y$ (where $X, Y$ are topological spaces) is almost continuous if, for each open set $U \subset X \times Y$ containing the graph of $f, U$ contains the graph of some continuous function $g: X \rightarrow Y$.

Almost continuity has become a property studied intensively by many mathematicians ([2], [9], [10]. Many results in the field of almost continuous functions are cited in the paper [12].) because almost continuous functions possess interesting topological properties, and many important classes of functions are connected with this property. (For example: every derivative or approximately continuous function is an almost continuous function.)

In the present paper we shall consider the properties of ideals of some rings of almost continuous functions, being extensions of rings of continuous functions.

Key Words: ring, ideal, essential ideal, prime ideal, porosity, Hausdorff metric.

Mathematical Reviews subject classification: 54C40,13A15, 26A15, 54C08

Received by the editors June 15, 1998 


\section{Preliminaries}

Throughout the paper, we shall consider functions $h: \mathbb{I} \rightarrow \mathbb{R}$ where $\mathbb{I}$ is a fixed closed interval or $\mathbb{I}=\mathbb{R}$, and $\mathbb{R}$ is the set of all real numbers with the natural topology.

The family of all almost continuous functions (continuous functions) will be denoted by $\mathcal{A},(\mathcal{C}){ }^{1}$

We shall use the standard notions and notation $([6],[7])$. The open ball with center at $x$ and radius $r>0$ will be denoted by $B(x, r)$. The symbols $\bar{A}, \operatorname{Fr}(A)$ and $\operatorname{Int}(A)$ stand for the closure, the boundary and the interior of $A$, respectively. The distance between a point $x$ and a set $A$ on the real line will be denoted by $\operatorname{dist}(x, A)$.

By $C_{\xi}\left(D_{\xi}\right)$ we shall denote the set of all continuity (discontinuity) points of $\xi$. The symbol $\Gamma(\xi)$ stands for the graph of $\xi$. $\mathcal{Z}(\xi)=\{x: \xi(x)=0\}$.

Let const $_{\alpha}$ denote the constant function assuming the value $\alpha$. For a function $\xi: \mathbb{I} \rightarrow \mathbb{R}$, let $\xi_{\alpha}^{\beta}$ denote a function defined in the following way:

$$
\xi_{\alpha}^{\beta}(x)=\left(\xi \wedge \operatorname{const}_{\beta}\right) \vee \text { const }_{\alpha}=\left\{\begin{array}{lll}
\beta & \text { if } & \xi(x) \geq \beta, \\
\xi(x) & \text { if } & \xi(x) \in[\alpha, \beta], \\
\alpha & \text { if } & \xi(x) \leq \alpha .
\end{array}\right.
$$

Let $\mathcal{F}$ be a fixed family of functions. Let us denote $([7]) \mathcal{Z}[\mathcal{F}]=\{\mathcal{Z}(\xi)$ : $\xi \in \mathcal{F}\}$ and by $\mathcal{F}_{b}$ the set $\left\{\xi_{\alpha}^{\beta}: \xi \in \mathcal{F} \wedge \alpha<0<\beta\right\}$ (as well as the metric space $\left(\mathcal{F}_{b}, \varrho\right)$ endowed with the metric of uniform convergence $\varrho$ ).

The notions and symbols we use, connected with porosity, come from papers [20] and [21]. Let $X$ be a metric space. Let $M \subset X, x \in X$ and $R>0$. Then we denote by $\gamma(x, R, M)$ the supremum of the set of all $r>0$ for which there exists $z \in X$ such that $B(z, r) \subset B(x, R) \backslash M$. If $p(M, x)=2 \cdot \lim \sup _{R \rightarrow 0+} \frac{\gamma(x, R, M)}{R}>0$, then we say that $M$ is porous at $x$. If there exists $\mu>0$ such that $p(M, z) \geq \mu$ for $z \in X$, then we say that $M$ is uniformly porous.

Let $\mathcal{D}$ denote the set of all closed subsets of $\mathbb{I} \neq \mathbb{R}$. Then by $\varrho_{H}^{\mathbb{I}}$ we shall denote the Hausdorff metric

$$
\varrho_{H}^{\mathbb{I}}(A, B)=\max \left(\sup _{a \in A}(\operatorname{dist}(a, B)), \sup _{b \in B}(\operatorname{dist}(b, A))\right) .
$$

Let $\mathcal{R}$ be a fixed ring. Then by $\Im(\mathcal{R})$ we shall denote the set of all ideals of $\mathcal{R}$. An ideal $\mathcal{J}_{1} \in \Im(\mathcal{R})$ will be called an extension (restriction) of a ideal $\mathcal{J}_{2} \in \Im(\mathcal{R})$ if $\mathcal{J}_{1} \supset \mathcal{J}_{2}\left(\mathcal{J}_{1} \subset \mathcal{J}_{2}\right)$.

\footnotetext{
${ }^{1}$ The domain and the range of functions from $\mathcal{A},(\mathcal{C})$ will always be obvious from the context.
} 
A nonzero ideal $\mathcal{J}_{o} \in \Im(\mathcal{R})$ is called essential ([1]) if it intersects every nonzero ideal nontrivially. The intersection of all essential ideals is called a socle. An ideal $\mathcal{J}$ is prime if $a b \in \mathcal{J}$ implies $a \in \mathcal{J}$ or $b \in \mathcal{J}$. An ideal $\mathcal{J} \in$ $\Im(\mathcal{R})$ is called a $z$-ideal if $\xi \in \mathcal{R}$ and $\mathcal{Z}(\xi) \in \mathcal{Z}[\mathcal{J}]$ implies $\xi \in \mathcal{J}$. Nontrivially, a z-ideal $\mathcal{J} \in \Im(\mathcal{R})$ will be called a $z^{\prime}$-ideal if $\bigcap \mathcal{Z}[\mathcal{J}]$ is a nonempty closed set belonging to $\mathcal{Z}[\mathcal{J}]$. If $\xi \in \mathcal{R}$, then the symbol $(\xi)_{\mathcal{R}}$ will stand for the ideal generated by $\xi$.

Let $\mathcal{R}$ be a fixed ring of functions. Then by $\operatorname{Ann}(A)$ we shall denote the set $\left\{\xi \in \mathcal{R}: \xi \cdot A=\left\{\right.\right.$ const $\left.\left._{0}\right\}\right\}(\operatorname{Ann}(A)$ is the set of all annihilators of $A)$.

A set $\left\{\mathcal{J}_{i}\right\}_{i \in I}$ of nonzero ideals in the rings of functions $\mathcal{R}$ is said to be independent if $\mathcal{J}_{i} \cap\left(\Sigma_{j \neq i} \mathcal{J}_{j}\right)=\left(\text { const }_{0}\right)_{\mathcal{R}}$, i.e. $\Sigma_{i \in I}=\bigoplus_{i \in I} \mathcal{J}_{i}$. Then we say that $\mathcal{R}$ has a finite Goldie dimension if every independent set of nonzero ideals is finite, and if $\mathcal{R}$ has no finite Goldie dimension, then the Goldie dimension of $\mathcal{R}$, denoted by $\operatorname{dim}(\mathcal{R})$, is the smallest cardinal number $m$ for which any independent set of nonzero ideals in $\mathcal{R}$ has cardinality less than or equal to $m$.

Let $f \in \mathcal{A}$ be a function such that $D_{f}=\overline{D_{f}} \subset \mathcal{Z}(f)^{2}$. By the symbol $\mathcal{C}_{\mathcal{A}}(f)$ we shall denote the set of all $\mathcal{A}$ - extensions rings of $\mathcal{C}$ containing $f$, i.e.

$$
\begin{array}{ll}
\text { a ring } \mathcal{R} \text { belongs to } \mathcal{C}_{\mathcal{A}}(f) \text { if and only if } & \mathcal{C} \cup\{f\} \subset \mathcal{R} \subset \mathcal{A} \\
& \text { and } D_{g} \subset D_{f}(g \in \mathcal{R}) .
\end{array}
$$

In the further considerations, if we write $\mathcal{C}_{\mathcal{A}}(f)$, then we always assume that $f$ is a fixed function belonging to $\mathcal{A}$, such that $\emptyset \neq D_{f}=\overline{D_{f}} \subset \mathcal{Z}(f)$.

Our considerations start with the observation that the results included in papers [2], [12], [13], [14], [15], [17] show that, for a function $f$ satisfying the above assumptions, $\mathcal{C}_{\mathcal{A}}(f) \neq \emptyset$ and, moreover, $\mathcal{C}_{\mathcal{A}}(f)$ contains more than one ring.

\section{Connections between Ideals of $\mathcal{C}$ and Ideals of $\mathcal{R} \in$ $\mathcal{C}_{\mathcal{A}}(f)$}

The first question that arises when one considers the set of rings $\mathcal{C}_{\mathcal{A}}(f)$ is connected with the possibility of the existence of rings $\mathcal{R}_{1}, \mathcal{R}_{2} \in \mathcal{C}_{\mathcal{A}}(f)$ for which the ideals $(f)_{\mathcal{R}_{1}},(f)_{\mathcal{R}_{2}}$ would be equal or distinct. The theorem below will show that there exist almost continuous functions as well as a continuum

\footnotetext{
${ }^{2}$ According to the results contained in paper [5], in this case, $D_{f}$ is a nowhere dense set and the family of all almost continuous functions fulfilling this condition is equal to the family of all Darboux functions fulfilling this condition.
} 
of rings $\mathcal{R}_{\eta} \in \mathcal{C}_{\mathcal{A}}(f)$ and a continuum of rings $\mathcal{K}_{\eta} \in \mathcal{C}_{\mathcal{A}}(f)$, such that $(f)_{\mathcal{R}_{\eta_{1}}}=$ $(f)_{\mathcal{R}_{\eta_{2}}}$ and $(f)_{\mathcal{K}_{\eta_{1}}} \neq(f)_{\mathcal{K}_{\eta_{2}}}$, for $\eta_{1} \neq \eta_{2}$.

Before we formulate the main theorem, we note that, according to the wellknown results included in [2], [3], [12], [19], the following Lemma is obvious.

Lemma 1. Let $f: \mathbb{I} \rightarrow \mathbb{R}$ be a function such that $D_{f}$ is a countable set, for which the following Young's condition is fulfilled.

$\forall_{x \in \mathbb{I}} \exists_{\left\{\alpha_{n}\right\},\left\{\beta_{n}\right\} \subset \mathbb{I}} \alpha_{n} \nearrow x \swarrow \beta_{n} \wedge \lim _{n \rightarrow \infty} f\left(\alpha_{n}\right)=f(x)=\lim _{n \rightarrow \infty} f\left(\beta_{n}\right)$. (Of course, if $x$ is an endpoint of $\mathbb{I}$, then there exists only one sequence.)

Then $f$ is an almost continuous function.

Theorem 1. For each countable and closed set $P \subset \mathbb{I}$, there exists a function $f: \mathbb{I} \rightarrow \mathbb{R}$ such that $f \in \mathcal{A}$ and $D_{f}=P$, for which there exist two families of rings $\left\{\mathcal{R}_{\eta}: \eta<\mathbf{c}\right\},\left\{\mathcal{K}_{\eta}: \eta<\mathbf{c}\right\} \subset \mathcal{C}_{\mathcal{A}}(f)$ such that $\mathcal{R}_{\eta_{1}} \neq \mathcal{R}_{\eta_{2}}, \mathcal{K}_{\eta_{1}} \neq \mathcal{K}_{\eta_{2}}$ $\left(\eta_{1} \neq \eta_{2}\right)$ and

(i) $(f)_{\mathcal{R}_{\eta_{1}}}=(f)_{\mathcal{R}_{\eta_{2}}}\left(\eta_{1}, \eta_{2}<\mathbf{c}\right)$;

(ii) $(f)_{\mathcal{K}_{\eta_{1}}} \neq(f)_{\mathcal{K}_{\eta_{2}}}\left(\eta_{1}, \eta_{2}<\mathbf{c}\right.$ and $\left.\eta_{1} \neq \eta_{2}\right)$.

Proof. If $\mathbb{I}$ is a bounded interval, then assume for simplicity that the endpoints of $\mathbb{I}$ belong to $P$. (In the opposite case, the proof is similar, but the notation would be more complicated.) Let $\left\{\left(a_{n}, b_{n}\right)\right\}_{n}$ be the sequence of all components of the complement of $P$. Fix $n_{0}$. Let $\left\{\beta_{k}^{n_{0}}\right\}\left(\left\{\gamma_{k}^{n_{0}}\right\}\right)$ be a sequence of numbers belonging to $\left(a_{n_{0}}, b_{n_{0}}\right)$ monotonically decreasing (increasing) to $a_{n_{0}}\left(b_{n_{0}}\right)$. (Additionally we can assume that $\beta_{1}^{n_{0}}<\gamma_{1}^{n_{0}}$.) Now, we choose $\alpha_{k}^{n_{0}} \in\left(\beta_{k+1}^{n_{0}}, \beta_{k}^{n_{0}}\right), \xi_{k}^{n_{0}} \in\left(\gamma_{k}^{n_{0}}, \gamma_{k+1}^{n_{0}}\right)$. Finally, let $c_{k}^{n_{0}}\left(d_{k}^{n_{0}}\right)$ be a mid point of the interval $\left(\beta_{k+1}^{n_{0}}, \alpha_{k}^{n_{0}}\right)\left(\left(\xi_{k}^{n_{0}}, \gamma_{k+1}^{n_{0}}\right)\right)$ for $k=1,2, \ldots$

Now, we define a function $f: \mathbb{I} \rightarrow \mathbb{R}$ by $f\left(c_{k}^{n}\right)=1=f\left(d_{k}^{n}\right)$ (for any $n$ and $k$ ); $f(x)=0$ for $x \in P, x \in\left[\alpha_{k}^{n}, \beta_{k}^{n}\right] \cup\left[\gamma_{k}^{n}, \xi_{k}^{n}\right]$ and $x \in\left[\beta_{1}^{n}, \gamma_{1}^{n}\right]$ (for any $n$ and $k$ ) and, moreover, let $f$ be linear in any interval $\left[\beta_{k+1}^{n}, c_{k}^{n}\right],\left[c_{k}^{n}, \alpha_{k}^{n}\right],\left[\xi_{k}^{n}, d_{k}^{n}\right]$, $\left[d_{k}^{n}, \gamma_{k+1}^{n}\right]$ (for any $n$ and $k$ ). It is easy to see that $P=D_{f} \subset \mathcal{Z}(f)$, and $f$ fulfills Young's condition, which means (Lemma 1) that $f \in \mathcal{A}$.

Now, we shall construct the families of rings $\left\{\mathcal{R}_{\eta}: \eta<\mathbf{c}\right\}$ and $\left\{\mathcal{K}_{\eta}: \quad \eta<\mathbf{c}\right\}$. Let $\left\{p_{\eta}^{n, k}\right\}_{\eta<\mathbf{c}}\left(\left\{q_{\eta}^{n, k}\right\}_{\eta<\mathbf{c}}\right)$ be the transfinite sequence of all points of the interval $\left(\alpha_{k}^{n}, \beta_{k}^{n}\right)\left(\left(\gamma_{k}^{n}, \xi_{k}^{n}\right)\right)$ for any $n, k$. Moreover, let for any $n, k, \delta_{k}^{n}>0$ be a real number such that $\left(\alpha_{k}^{n}-\delta_{k}^{n}, \alpha_{k}^{n}\right) \subset\left(c_{k}^{n}, \alpha_{k}^{n}\right)$, $\left(\gamma_{k}^{n}-\delta_{k}^{n}, \gamma_{k}^{n}\right) \subset\left(d_{k-1}^{n}, \gamma_{k}^{n}\right)$ (we put $\left.\left(d_{0}, \gamma_{1}^{n}\right)=\left\{\gamma_{1}^{n}\right\}\right)$ and $f\left(\left[\alpha_{k}^{n}-\delta_{k}^{n}, \alpha_{k}^{n}\right]\right) \subset$ $\left(-\frac{1}{k}, \frac{1}{k}\right) \supset f\left(\left[\gamma_{k}^{n}-\delta_{k}^{n}, \gamma_{k}^{n}\right]\right)$. Let $\left\{u_{\eta}^{n, k}\right\}_{\eta<\mathbf{c}}\left(\left\{v_{\eta}^{n, k}\right\}_{\eta<\mathbf{c}}\right)$ be the transfinite sequence of all points of $\left(\alpha_{k}^{n}-\delta_{k}^{n}, \alpha_{k}^{n}\right)\left(\left(\gamma_{k}^{n}-\delta_{k}^{n}, \gamma_{k}^{n}\right)\right)$.

For a fixed $\eta$, we denote by $\mathcal{R}_{\eta}$ the ring of all functions $\varphi: \mathbb{I} \rightarrow \mathbb{R}$ such that:

(a) $D_{\varphi} \subset P$, 
(b) $\varphi_{\mid A_{\eta}}$ is a continuous function, where $A_{\eta}=P \cup \bigcup_{n, k}\left\{p_{\eta}^{n, k}, q_{\eta}^{n, k}\right\}$.

Moreover, by $\mathcal{K}_{\eta}$ we shall denote the ring of all functions $\psi: \mathbb{I} \rightarrow \mathbb{R}$ such that: (A) $D_{\psi} \subset P$,

(B) $\psi_{\mid B_{\eta}}$ is a continuous function, where $B_{\eta}=P \cup \bigcup_{n, k}\left\{u_{\eta}^{n, k}, v_{\eta}^{n, k}\right\}$.

Of course, $f \in \mathcal{R}_{\eta}(\eta<\mathbf{c})$ and, moreover, according to our assumptions and Lemma $1, \varphi \in \mathcal{A}$ for any $\varphi \in \mathcal{R}_{\eta}(\eta<\mathbf{c})$. Consequently, it is easy to see that $\mathcal{R}_{\eta} \in \mathcal{C}_{\mathcal{A}}(f)(\eta<\mathbf{c})$. Clearly, if $\eta_{1} \neq \eta_{2}\left(\eta_{1}, \eta_{2}<\mathbf{c}\right)$, then $\mathcal{R}_{\eta_{1}} \neq \mathcal{R}_{\eta_{2}}$.

In a similar way as in the case of $\mathcal{R}_{\eta}$, we can observe that $\mathcal{K}_{\eta} \in \mathcal{C}_{\mathcal{A}}(f)$ $(\eta<\mathbf{c})$, and if $\eta_{1} \neq \eta_{2}\left(\eta_{1}, \eta_{2}<\mathbf{c}\right)$, then $\mathcal{K}_{\eta_{1}} \neq \mathcal{K}_{\eta_{2}}$.

Now, we shall show that

$$
(f)_{\mathcal{R}_{\eta_{1}}}=(f)_{\mathcal{R}_{\eta_{2}}} \text { for } \eta_{1} \neq \eta_{2}\left(\eta_{1}, \eta_{2}<\mathbf{c}\right)
$$

More precisely, we shall prove only the inclusion $(f)_{\mathcal{R}_{\eta_{1}}} \subset(f)_{\mathcal{R}_{\eta_{2}}}$ because the proof of the inclusion $(f)_{\mathcal{R}_{\eta_{1}}} \supset(f)_{\mathcal{R}_{\eta_{2}}}$ is similar. Let $t=f_{1} \cdot f \in(f)_{\mathcal{R}_{\eta_{1}}}$ $\left(f_{1} \in \mathcal{R}_{\eta_{1}}\right)$, and for any $n$, $k$, let $\left(r_{1}^{n, k}, r_{2}^{n, k}\right)\left(\left(w_{1}^{n, k}, w_{2}^{n, k}\right)\right)$ be an interval contained in $\left(\alpha_{k}^{n}, \beta_{k}^{n}\right)\left(\left(\gamma_{k}^{n}, \xi_{k}^{n}\right)\right)$, such that $p_{\eta_{1}}^{n, k} \notin\left(r_{1}^{n, k}, r_{2}^{n, k}\right) \ni p_{\eta_{2}}^{n, k}\left(q_{\eta_{1}}^{n, k} \notin\right.$ $\left.\left(w_{1}^{n, k}, w_{2}^{n, k}\right) \ni q_{\eta_{2}}^{n, k}\right)$. Now, we define a function $f_{2}: \mathbb{I} \rightarrow \mathbb{R}$ in the following way:

$$
f_{2}(x)= \begin{cases}f_{1}(x) & \text { for } x \notin \bigcup_{n, k}\left(\left(r_{1}^{n, k}, r_{2}^{n, k}\right) \cup\left(w_{1}^{n, k}, w_{2}^{n, k}\right)\right) \text { and } x \in A_{\eta_{2}} \\ 0 & \text { for } x \in \bigcup_{n, k}\left\{p_{\eta_{2}}^{n, k}, q_{\eta_{2}}^{n, k}\right\} \\ \text { linear } & \text { in each segment }\left[r_{1}^{n, k}, p_{\eta_{2}}^{n, k}\right],\left[p_{\eta_{2}}^{n, k}, r_{2}^{n, k}\right],\left[w_{1}^{n, k}, q_{\eta_{2}}^{n, k}\right] \\ & \text { and }\left[q_{\eta_{2}}^{n, k}, w_{2}^{n, k}\right] .\end{cases}
$$

It is easy to see that $f \in \mathcal{R}_{\eta_{2}}$ and $t=f_{2} \cdot f \in(f)_{\mathcal{R}_{\eta_{2}}}$, which ends the proof of (1).

The proof is completed by showing that

$$
(f)_{\mathcal{K}_{\eta_{1}}} \neq(f)_{\mathcal{K}_{\eta_{2}}} \text { for } \eta_{1} \neq \eta_{2}\left(\eta_{1}, \eta_{2}<\mathbf{c}\right)
$$

For any $n, k$, let $\left(z_{1}^{n, k}, z_{2}^{n, k}\right)\left(\left(y_{1}^{n, k}, y_{2}^{n, k}\right)\right)$ be an interval such that its closure is contained in $\left(\alpha_{k}^{n}-\delta_{k}^{n}, \alpha_{k}^{n}\right)\left(\left(\gamma_{k}^{n}-\delta_{k}^{n}, \gamma_{k}^{n}\right)\right)$ and $u_{\eta_{1}}^{n, k} \notin\left(z_{1}^{n, k}, z_{2}^{n, k}\right) \ni u_{\eta_{2}}^{n, k}$, $\left(v_{\eta_{1}}^{n, k} \notin\left(y_{1}^{n, k}, y_{2}^{n, k}\right) \ni v_{\eta_{2}}^{n, k}\right)$. Now, we define a function $d: \mathbb{I} \rightarrow \mathbb{R}$ by

$$
d(x)= \begin{cases}0 & \text { for } x \notin \cup_{n, k}\left(\left(z_{1}^{n, k}, z_{2}^{n, k}\right) \cup\left(y_{1}^{n, k}, y_{2}^{n, k}\right)\right) \\ \frac{k}{f(x)} & \text { for } x=u_{\eta_{2}}^{n, k} \text { or } x=v_{\eta_{2}}^{n, k}(\text { for any } n, k) \\ \text { linear } & \text { in any segment }\left[z_{1}^{n, k}, u_{\eta_{2}}^{n, k}\right],\left[u_{\eta_{2}}^{n, k}, z_{2}^{n, k}\right],\left[y_{1}^{n, k}, v_{\eta_{2}}^{n, k}\right] \\ & \text { and }\left[v_{\eta_{2}}^{n, k}, y_{2}^{n, k}\right](\text { for any } n, k) .\end{cases}
$$


It is easy to see that $d \in \mathcal{K}_{\eta_{1}}$. Put $d_{0}=d \cdot f \in(f)_{\mathcal{K}_{\eta_{1}}}$. Observe that $d_{0} \notin \mathcal{K}_{\eta_{2}}$, which ends the proof of (2).

In many papers and monographs (e.g. [1], [7], [8]) the authors investigated the ideals of rings of continuous functions (often defined on more abstract spaces than $\mathbb{R}$ ). So, to begin with, let us note the relations between ideals of the rings of continuous functions and ideals of rings belonging to $\mathcal{C}_{\mathcal{A}}(f)$. We make some preliminary observations.

Remark. For an arbitrary ring $\mathcal{R} \in \mathcal{C}_{\mathcal{A}}(f)$, there exists an ideal $\mathcal{J}_{0}$ of the ring $\mathcal{C}$ (of all continuous functions) which is not an ideal of $\mathcal{R}$.

In fact. Let $[a, b]$ be a nondegenerate interval such that $[a, b] \cap D_{f}=\emptyset$ and let $x_{0} \in(a, b)$. Put $\mathcal{J}_{0}=\left\{h \in \mathcal{C}: h\left(x_{0}\right)=0\right\}$. Thus $\mathcal{J}_{0} \in \Im(\mathcal{C})$. Now, we consider a function $k: \mathbb{I} \rightarrow \mathbb{R}$ defined by

$$
k(x)= \begin{cases}0 & \text { for } x=x_{0} \\ 1 & \text { for } x \notin[a, b], \\ \text { linear } & \text { in the segments }\left[a, x_{0}\right] \text { and }\left[x_{0}, b\right] .\end{cases}
$$

Note that $k \in \mathcal{J}_{0}$, but $f \cdot k \notin \mathcal{J}_{0}$.

Of course, it is easy to see that there exists an ideal $\mathcal{J} \in \Im(\mathcal{C})$ such that $\mathcal{J} \in \Im(\mathcal{R})$ where $\mathcal{R}$ is some ring belonging to $\mathcal{C}_{\mathcal{A}}(f)$. So, the following question seems to be interesting. Let $\mathcal{R} \in \mathcal{C}_{\mathcal{A}}(f)$. Under what additional assumptions connected with an ideal $\mathcal{J} \in \Im(\mathcal{C})$ does there exists an extension $\mathcal{J}^{*}$ (restriction $\left.\mathcal{J}_{*}\right)$ of $\mathcal{J}$ such that $\mathcal{J}^{*} \in \Im(\mathcal{C}) \backslash \Im(\mathcal{R})\left(\mathcal{J}_{*} \in \Im(\mathcal{C}) \cap \Im(\mathcal{R})\right)$ ? The answer is included in the theorem below. Before giving this theorem we can formulate the following Lemma.

Lemma 2. Let $\mathcal{J}_{0}$ be an ideal of $\mathcal{C}$ which is an ideal of $\mathcal{R} \in \mathcal{C}_{\mathcal{A}}(f)$. Then $D_{f} \subset \cap \mathcal{Z}\left[\mathcal{J}_{0}\right]$.

Proof. Suppose the Lemma were false. Then there is $x_{0} \in D_{f} \backslash \cap \mathcal{Z}\left[\mathcal{J}_{0}\right]$. Let $h \in \mathcal{J}_{0}$ be a function such that $h\left(x_{0}\right)=\alpha \neq 0$. There is no loss of generality in assuming that $\alpha>0$. Since $x_{0} \in D_{f}=\overline{D_{f}} \subset \mathcal{Z}(f)$, and $f$ is an almost continuous function, there exists a sequence $\left\{x_{n}\right\}_{n=1}^{\infty}$ such that $x_{n} \rightarrow x_{0}$ and $f\left(x_{n}\right) \rightarrow \beta \neq 0$. Put $\gamma=\min (\alpha,|\beta|)$. Without loss of generality we may assume that $\left|f\left(x_{n}\right)\right|>\frac{\gamma}{2}(n=1,2, \ldots)$ and $h\left(x_{n}\right)>\frac{\gamma}{2}$. Let $k=f \cdot h$. It is easy to verify that $k\left(x_{n}\right)$ does not converge to $k\left(x_{0}\right)=0$, which means that $\mathcal{J}_{0} \notin \Im(\mathcal{R})$. The contradiction obtained ends the proof of the Lemma.

Theorem 2. Let $\mathcal{R} \in \mathcal{C}_{\mathcal{A}}(f)$. For an arbitrary $z^{\prime}$-ideal $\mathcal{J} \in \Im(\mathcal{C})$ for which $A=\bigcap \mathcal{Z}[\mathcal{J}]$ is not a singleton, there exist: 
(A) an extension $\mathcal{J}^{*}$ of $\mathcal{J}$ which is a $z^{\prime}$-ideal of $\mathcal{C}$, such that $\mathcal{J}^{*} \notin \Im(\mathcal{R})$,

(B) a restriction $\mathcal{J}_{*}$ of $\mathcal{J}$ which is a $z^{\prime}$-ideal of $\mathcal{C}$, such that $\mathcal{J}_{*} \in \Im(\mathcal{R})$.

Proof. If $\mathcal{J}$ is not an ideal in $\mathcal{R}$, then we put $\mathcal{J}^{*}=\mathcal{J}$. Otherwise, let $x_{0} \in D_{f}$. Since $A$ is not a singleton, there exists $\delta>0$ such that $A^{\prime}=$ $A \backslash\left(x_{0}-\delta_{0}, x_{0}+\delta_{0}\right) \neq \emptyset$. Let us assume $\mathcal{J}^{*}=\left\{g \in \mathcal{C}: g\left(A^{\prime}\right)=\{0\}\right\}$. Of course, $\mathcal{J}^{*}$ is a $\mathrm{z}^{\prime}$-ideal of $\mathcal{C}$ and $\mathcal{J} \subset \mathcal{J}^{*}$. Since $D_{f}$ is not included in $\bigcap \mathcal{Z}\left[\mathcal{J}^{*}\right]=A^{\prime}$, according to Lemma $2, \mathcal{J}^{*}$ is not an ideal of $\mathcal{R}$.

Now, we are going to prove $(B)$. If $\mathcal{J}$ is an ideal of $\mathcal{R}$, then we put $\mathcal{J}_{*}=\mathcal{J}$. In the opposite case, there exists a point $p \notin D_{f} \cup A$. ( $A$ is a closed set different from the real line and $D_{f}$ is a nowhere dense set.) Let $\varepsilon>0$ be a real number such that $(p-\varepsilon, p+\varepsilon) \cap\left(D_{f} \cup A\right)=\emptyset$. Let us denote by $\mathcal{J}_{*}$ the family $\{g \in \mathcal{J}: \mathbb{R} \backslash(p-\varepsilon, p+\varepsilon) \subset \mathcal{Z}(g)\}$. It is easy to check that $J_{*}$ is a $\mathrm{z}^{\prime}$-ideal of $\mathcal{C}$.

Now, we shall show that $\mathcal{J}_{*}$ is an ideal of $\mathcal{R}$. Of course, the sum of arbitrary functions from $\mathcal{J}_{*}$ belongs to $\mathcal{J}_{*}$. So, let $k \in \mathcal{R}$ and $g \in \mathcal{J}_{*}$. Let us define

$$
k_{0}(x)= \begin{cases}k(x) & \text { for } \quad x \in(p-\varepsilon, p+\varepsilon) \\ k(p-\varepsilon) & \text { for } \quad x \leq p-\varepsilon \\ k(p+\varepsilon) & \text { for } \quad x \geq p+\varepsilon\end{cases}
$$

Note that $k \cdot g=k_{0} \cdot g \in \mathcal{J}$ and $\mathcal{Z}(k \cdot g) \supset(-\infty, p-\varepsilon] \cup[p+\varepsilon,+\infty)$, which means that $k \cdot g \in \mathcal{J}_{*}$.

Now, we shall consider the following question. We have a fixed ideal $\mathcal{J}$ of $\mathcal{R} \in \mathcal{C}_{\mathcal{A}}(f)$. Does there exist a restriction $\mathcal{J}_{*}$ of $\mathcal{J}$ such that $\mathcal{J}_{*}$ is an ideal of $\mathcal{C}$ and $\mathcal{R}$ ? The following theorem gives the answer to this question.

Theorem 3. Let $f$ be a function for which $D_{f}$ is a countable set. For each ideal $\mathcal{J}$ of $\mathcal{R} \in \mathcal{C}_{\mathcal{A}}(f)$, there exists a restriction $\mathcal{J}_{*} \in \Im(\mathcal{C}) \cap \Im(\mathcal{R})$. Moreover, if $\mathcal{J}$ is an essential ideal of $\mathcal{R}$, then we may assume that $\mathcal{J}_{*}$ is also an essential ideal of $\mathcal{R}$.

Proof. Let $\left\{q_{n}\right\}_{n}$ be a sequence of all points of the set $D_{f}$ and let $\varepsilon_{0}>0$ be a real number such that $\bigcup_{n}\left(q_{n}-\varepsilon_{0}, q_{n}+\varepsilon_{0}\right) \neq \mathbb{R}$. For an arbitrary $\varepsilon \in\left(0, \varepsilon_{0}\right)$, let

$$
A_{\varepsilon}(f)=\bigcup_{n}\left(q_{n}-\varepsilon, q_{n}+\varepsilon\right) \text {. }
$$

Let us put $\mathcal{J}^{\prime}=\left\{h \in \mathcal{C}: \exists_{\varepsilon \in\left(0, \varepsilon_{0}\right)} A_{\varepsilon}(f) \subset \mathcal{Z}(h)\right\}$. It follows immediately that

$$
\mathcal{J}^{\prime} \in \Im(\mathcal{C}) \cap \Im(\mathcal{R})
$$

This means that $\mathcal{J}_{*}=\mathcal{J}^{\prime} \cap \mathcal{J} \in \Im(\mathcal{C}) \cap \Im(\mathcal{R})$. 
Now, we additionally assume that $\mathcal{J}$ is an essential ideal of $\mathcal{R}$. Let $\mathcal{J}_{0}$ be an arbitrary ideal of $\mathcal{R}$. Of course, $\mathcal{J}$ intersects $\mathcal{J}_{0}$ nontrivially. So, let $k \in \mathcal{J} \cap \mathcal{J}_{0}$ be a function for which some $x_{0}$ is an element such that $k\left(x_{0}\right) \neq 0$. We assume, for instance, that $k\left(x_{0}\right)>0$. Since $k$ is a Darboux function, $x_{0}$ is a Darboux point of $k([4],[11])$. Thus there exists a point $y_{0}$ such that $k\left(y_{0}\right)>0$, $y_{0} \notin D_{f}$. Let $\delta_{0}>0$ be a real number such that $\left[y_{0}-\delta_{0}, y_{0}+\delta_{0}\right] \cap D_{f}=\emptyset$. Define a function $h_{0}: \mathbb{I} \rightarrow \mathbb{R}$ by

$$
h_{0}(x)= \begin{cases}0 & \text { for } x \leq y_{0}-\delta_{0} \text { or } x \geq y_{0}+\delta_{0}, \\ 1 & \text { for } x=y_{0}, \\ \text { linear } & \text { in the intervals }\left[y_{0}-\delta_{0}, y_{0}\right] \text { and }\left[y_{0}, y_{0}+\delta_{0}\right]\end{cases}
$$

Then $A_{\varepsilon^{\prime}}(f) \subset \mathcal{Z}\left(h_{0}\right)$, for some $\varepsilon^{\prime} \in\left(0, \varepsilon_{0}\right)$, and so, $h_{0} \in \mathcal{J}^{\prime}$. Let $h_{*}=h_{0} \cdot k \neq$ const $_{0}$. It is not hard to verify that $h_{*} \in \mathcal{J}_{*} \cap \mathcal{J}_{0}$.

\section{The Properties of Ideals of $\mathcal{R} \in \mathcal{C}_{\mathcal{A}}(f)$}

In the next theorem we discuss the following problem. For a fixed ideal $\mathcal{J} \in$ $\mathcal{R} \in \mathcal{C}_{\mathcal{A}}(f)$ does there exist a "small" (in the topological sense) set $A \subset \mathcal{R}$ such that $\mathcal{J} \oplus A$ is an essential ideal of $\mathcal{R}$ ?

Theorem 4. Let $\mathcal{J}$ be a nontrivial ideal of rings $\mathcal{R} \in \mathcal{C}_{\mathcal{A}}(f)$. Then the set $A=\operatorname{Ann}(\mathcal{J})$ possesses the following property: $A_{b}$ is uniformly porous (in $\mathcal{R}_{b}$ ) and $\mathcal{J} \oplus A$ is an essential ideal of $\mathcal{R}$.

Proof. Of course, $\mathcal{J} \oplus A$ is an essential ideal of $\mathcal{R}$. Now, we shall prove that $A_{b}$ is a uniformly porous set in $\mathcal{R}_{b}$. Let $h \in \mathcal{R}_{b}$ and let $\varepsilon>0$. Fix a nonzero function $g \in \mathcal{J}$ and let $z_{0}$ be a point such that $g\left(z_{0}\right) \neq 0$. For the proof, we analyze the possible cases:

$1^{o} 0 \leq h\left(z_{0}\right)<\frac{\varepsilon}{2}$. Let $\xi=h+$ const $_{\frac{\varepsilon}{2}} \in \mathcal{R}_{b}$ and consider the open ball (in the space $\left.\mathcal{R}_{b}\right) B\left(\xi, \frac{\varepsilon}{4}\right)$. Note that $B\left(\xi, \frac{\varepsilon}{4}\right) \cap A_{b}=\emptyset$. In fact, suppose to the contrary that there exists $\varphi \in B\left(\xi, \frac{\varepsilon}{4}\right) \cap A_{b}$. Thus $\varphi\left(z_{0}\right) \neq 0$. Let $\psi \in A$ be a function such that $\varphi=\psi_{\alpha}^{\beta}$ for some $\alpha, \beta(\alpha<0<\beta)$. Clearly, $\psi\left(z_{0}\right) \neq 0$. According to the inequality $\psi \cdot g\left(z_{0}\right) \neq 0$ we have $\psi \notin A$. It is not hard to verify that $B\left(\xi, \frac{\varepsilon}{4}\right) \subset B(h, \varepsilon)$.

$2^{o}\left|h\left(z_{0}\right)\right| \geq \frac{\varepsilon}{2}$. Put $\xi=h$. Similarly as above we can prove that $B\left(\xi, \frac{\varepsilon}{4}\right) \cap$ $A_{b}=\emptyset$ and $B\left(\xi, \frac{\varepsilon}{4}\right) \subset B(h, \varepsilon)$.

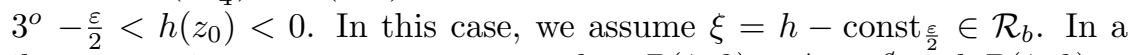
similar way as in case $1^{o}$ we can prove that $B\left(\xi, \frac{\varepsilon}{4}\right) \cap A_{b}=\emptyset$ and $B\left(\xi, \frac{\varepsilon}{4}\right) \subset$ $B(h, \varepsilon)$. 
The above considerations prove that $p\left(A_{b}, h\right) \geq \frac{1}{4}$, which, according to the arbitrariness of $h$, ends the proof.

Before we formulate the next properties we introduce a bit more notation. Let $\mathcal{R}_{0}$ be a fixed ring of functions mapping the interval $[0,1]$ into $\mathbb{R}$, such that $\mathcal{R}_{0} \in \mathcal{C}_{\mathcal{A}}(f)$. By $\Im_{z^{\prime}}\left(\mathcal{R}_{0}\right)$ we shall denote the set of all $z^{\prime}$-ideals of $\mathcal{R}_{0}$.

Proposition 1. Let $\mathcal{J}_{1}, \mathcal{J}_{2} \in \Im_{z^{\prime}}\left(\mathcal{R}_{0}\right)$. Then $\mathcal{J}_{1}=\mathcal{J}_{2}$ if and only if $\cap \mathcal{Z}\left[\mathcal{J}_{1}\right]=$ $\bigcap \mathcal{Z}\left[\mathcal{J}_{2}\right]$.

Proof. We only need to prove the sufficiency. Let $A=\bigcap \mathcal{Z}\left[\mathcal{J}_{1}\right]=\bigcap \mathcal{Z}\left[\mathcal{J}_{2}\right]$. First, we shall show that $\mathcal{J}_{1} \subset \mathcal{J}_{2}$. Let $f_{1} \in \mathcal{J}_{1}$. Then $A \subset \mathcal{Z}\left(f_{1}\right)$. Moreover, there exists $f_{2} \in \mathcal{J}_{2}$ such that $A=\mathcal{Z}\left(f_{2}\right)$. Thus

$$
\mathcal{J}_{2} \ni f_{1} \cdot f_{2} \text { and } \mathcal{Z}\left(f_{1} \cdot f_{2}\right)=\mathcal{Z}\left(f_{1}\right) \cup A=\mathcal{Z}\left(f_{1}\right) \text {, }
$$

which means that $\mathcal{Z}\left(f_{1}\right) \in \mathcal{Z}\left[\mathcal{J}_{2}\right]$ and, consequently, $f_{1} \in \mathcal{J}_{2}$. In a similar way we prove that $\mathcal{J}_{2} \subset \mathcal{J}_{1}$.

On the basis of the above Proposition, we can the set $\Im_{z^{\prime}}\left(\mathcal{R}_{0}\right)$ endowed with the metric $\varrho_{0}\left(\mathcal{J}_{1}, \mathcal{J}_{2}\right)=\varrho_{H}^{[0,1]}\left(\bigcap \mathcal{Z}\left[\mathcal{J}_{1}\right], \bigcap \mathcal{Z}\left[\mathcal{J}_{2}\right]\right)$.

Proposition 2. If $\mathcal{J} \in \Im_{z^{\prime}}\left(\mathcal{R}_{0}\right)$, then $\mathcal{J}$ is an intersection of prime ideals.

The proof is similar to that of Theorem 2.8 from [7] for rings of continuous functions.

It is not hard to give an example of a $z^{\prime}$-ideal which is not simple. So, the question arises whether this phenomenon is rare or frequent. The successive theorem will show that a "typical" 3 (in the topological sense) z'-ideal is not a prime ideal.

Theorem 5. Let $\mathcal{P}$ be the set of all prime ideals of a ring $\mathcal{R}_{0}$. Then $\mathcal{P} \cap$ $\Im_{z^{\prime}}\left(\mathcal{R}_{0}\right)$ is uniformly porous set in the space $\left(\Im_{z^{\prime}}\left(\mathcal{R}_{0}\right), \varrho_{0}\right)$.

Proof. Let $\mathcal{J} \in \Im_{z^{\prime}}\left(\mathcal{R}_{0}\right)$. Throughout the proof, $A$ denotes the intersection $\bigcap \mathcal{Z}[\mathcal{J}]$. Of course, $A \neq \emptyset$.

We shall consider two cases:

I) There exist real numbers $a$ and $b$ such that $a<b$ and $[a, b] \subset \operatorname{Int}(A)$.

Let $\delta>0$ be a real number such that $[a-\delta, b+\delta] \subset A$ and $b-a>2 \delta$. Let us first prove

$$
\forall_{\varepsilon_{0} \in(0, \delta)} \exists_{\mathcal{J}_{0} \in \Im_{z^{\prime}}\left(\mathcal{R}_{0}\right)} B\left(\mathcal{J}_{0}, \frac{\varepsilon_{0}}{5}\right) \cap \mathcal{P}=\emptyset
$$
$[4])$.

${ }^{3}$ The notion "typical" was considered in a lot of papers and monographs (see, for example, 
We choose points $p, q \in(a, b)$ such that

$$
\frac{\varepsilon_{0}}{2}<q-p<\varepsilon_{0}
$$

and put $A_{0}=A \backslash(p, q)$. Then let

$$
\mathcal{J}_{0}=\left\{g \in \mathcal{R}_{0}: A_{0} \subset \mathcal{Z}(g)\right\} .
$$

It is easy to observe that $\mathcal{J}_{0} \in \Im_{z^{\prime}}\left(\mathcal{R}_{0}\right)$ and $\varrho_{0}\left(\mathcal{J}_{0}, \mathcal{J}\right)<\frac{\varepsilon_{0}}{2}$. In the space $\left(\Im_{z^{\prime}}\left(\mathcal{R}_{0}\right), \varrho_{0}\right)$ let us consider an open ball $B\left(\mathcal{J}_{0}, \frac{\varepsilon_{0}}{5}\right)$. Note that $B\left(\mathcal{J}_{0}, \frac{\varepsilon_{0}}{5}\right) \subset$ $B(\mathcal{J}, \varepsilon)$.

Finally, let us prove that $B\left(\mathcal{J}_{0}, \frac{\varepsilon_{0}}{5}\right) \cap \mathcal{P}=\emptyset$. So, let $\mathcal{J}_{1} \in B\left(\mathcal{J}_{0}, \frac{\varepsilon_{0}}{5}\right)$. From $\varrho_{0}\left(\mathcal{J}_{0}, \mathcal{J}_{1}\right)<\frac{\varepsilon_{0}}{5}$ we conclude that $\mathcal{Z}\left[\mathcal{J}_{1}\right] \cap\left(p+\frac{\varepsilon_{0}}{5}, q-\frac{\varepsilon_{0}}{5}\right)=\emptyset$. According to (4), we infer that $\left(p+\frac{\varepsilon_{0}}{5}, q-\frac{\varepsilon_{0}}{5}\right)$ is a nondegenerate interval. Put

$$
\alpha=\sup \left\{y \in \bigcap \mathcal{Z}\left[\mathcal{J}_{1}\right]: y<p+\frac{\varepsilon_{0}}{5}\right\} ; \beta=\inf \left\{y \in \bigcap \mathcal{Z}\left[\mathcal{J}_{1}\right]: y>q-\frac{\varepsilon_{0}}{5}\right\} .
$$

Of course, $\alpha<\beta$. Now, consider nonnegative continuous functions $\varphi$ and $\psi$ such that $\mathcal{Z}(\varphi)=(-\infty, \alpha]$ and $\mathcal{Z}(\psi)=[\beta,+\infty)$. Moreover, let $\xi \in \mathcal{J}_{1} \in$ $\Im_{z^{\prime}}\left(\mathcal{R}_{0}\right)$ be a function such that $\mathcal{Z}(\xi)=\bigcap \mathcal{Z}\left[\mathcal{J}_{1}\right], \varphi_{0}=\xi^{2}+\varphi$ and $\psi_{0}=\xi^{2}+\psi$. Note that

$$
\mathcal{Z}\left(\varphi_{0}\right)=\bigcap \mathcal{Z}\left[\mathcal{J}_{1}\right] \cap(-\infty, \alpha] ; \mathcal{Z}\left(\psi_{0}\right)=\bigcap \mathcal{Z}\left[\mathcal{J}_{1}\right] \cap[\beta,+\infty) .
$$

The last observation leads to the equality $\mathcal{Z}\left(\varphi_{0} \cdot \psi_{0}\right)=\bigcap \mathcal{Z}\left[\mathcal{J}_{1}\right]$, which means that $\varphi_{0} \cdot \psi_{0} \in \mathcal{J}_{1}$. Of course, $\varphi_{0}, \psi_{0} \notin \mathcal{J}_{1}$. The proof of (3) is finished. From (3) we deduce that $p\left(\mathcal{P} \cap \Im_{z^{\prime}}\left(\mathcal{R}_{0}\right), \mathcal{J}\right) \geq \frac{2}{5}$.

II) $\operatorname{Int}(A)=\emptyset$. In this case, $A$ is a closed and nowhere dense set. Let $(a, b)$ be a fixed component of the complement of $A$. Assume that $a>-\infty$, then $a \in A$. Let $\delta>0$ be a real number such that $(a, a+2 \delta) \cap A=\emptyset$. Consider the relation

$$
\forall_{\varepsilon_{0} \in(0, \delta)} \exists_{\mathcal{J}_{0} \in \Im_{z^{\prime}}\left(\mathcal{R}_{0}\right)} B\left(\mathcal{J}_{0}, \frac{\varepsilon_{0}}{5}\right) \cap \mathcal{P}=\emptyset .
$$

To prove this fact, we put $q=a+\frac{\varepsilon_{0}}{2}$ and $\mathcal{J}_{0}=\left\{g \in \mathcal{R}_{0}: A \cup\{q\} \subset \mathcal{Z}(g)\right\}$. Then the proof of (5) is analogous to that of (3) from part I and, consequently, also in this case, $p\left(\mathcal{P} \cap \Im_{z^{\prime}}\left(\mathcal{R}_{0}\right), \mathcal{J}\right) \geq \frac{2}{5}$.

Finally, we shall consider a Goldie dimension of an arbitrary ring $\mathcal{R} \in$ $\mathcal{C}_{\mathcal{A}}(f)$.

Theorem 6. For an arbitrary $\mathcal{R} \in \mathcal{C}_{\mathcal{A}}(f), \operatorname{dim}(\mathcal{R})=\aleph_{0}$. 
Proof. Our proof starts with the observation that

$$
\operatorname{dim}(\mathcal{R}) \leq \aleph_{0}
$$

In fact, let $\bigoplus_{s \in S} \mathcal{J}_{s}$ be a direct sum of ideals in $\mathcal{R}$. To prove (6), it is sufficient to show that $\operatorname{card}(S) \leq \aleph_{0}$. Choose from each $\mathcal{J}_{s}(s \in S)$ a function $f_{s} \neq$ const $_{0}$ and denote $D_{s}^{+}=\operatorname{Int}\left(\mathbb{I} \backslash \mathcal{Z}\left(f_{s}\right)\right) \neq \emptyset(s \in S)$. Note that

$$
D_{s_{1}}^{+} \cap D_{s_{2}}^{+}=\emptyset \text { for } s_{1} \neq s_{2}
$$

Indeed, fix $s_{1}, s_{2} \in S$. Since $\mathcal{J}_{s_{1}} \cap \mathcal{J}_{s_{2}}=\left(\text { const }_{0}\right)_{\mathcal{R}}$, therefore $f_{s_{1}} \cdot f_{s_{2}}=$ const $_{0}$, which proves $(7)$.

From $(7)$ and the definition of $D_{s}^{+}$we infer that card $(S) \leq \aleph_{0}$, which ends the proof of $(6)$.

Now, we shall show that

$$
\operatorname{dim}(\mathcal{R}) \geq \aleph_{0}
$$

Let $\left\{\left(a_{n}, b_{n}\right)\right\}_{n=1}^{\infty}$ be a sequence of pairwise disjoint intervals such that $D_{f} \cap$ $\left(a_{n}, b_{n}\right)=\emptyset(n=1,2, \ldots)$. Denote by $q_{n}$ a middle point of the interval $\left(a_{n}, b_{n}\right)(n=1,2 \ldots)$ and for any $n$ let $\delta_{n}>0$ be a real number such that $\left[q_{n}-\delta_{n}, q_{n}+\delta_{n}\right] \subset\left(a_{n}, b_{n}\right)$.

Now, we shall define functions $g_{n}(n=1,2, \ldots)$ by

$$
g_{n}(x)= \begin{cases}0 & \text { for } x \notin\left(q_{n}-\delta_{n}, q_{n}+\delta_{n}\right), \\ 1 & \text { for } x=q_{n}(n=1,2, \ldots), \\ \text { linear } & \text { in each of the intervals }\left[q_{n}-\delta_{n}, q_{n}\right] \text { and }\left[q_{n}, q_{n}+\delta_{n}\right]\end{cases}
$$

It is easy to see that $g_{n} \in \mathcal{C} \subset \mathcal{R}$.

Now, we shall consider the family $\left\{\left(g_{n}\right)_{\mathcal{R}}: n=1,2, \ldots\right\}$ and show that it is a family of independent ideals. Suppose that, for some $n_{0}$, there exists $h \in \mathcal{R}$ such that

$$
h \in\left(g_{n_{0}}\right)_{\mathcal{R}} \cap \Sigma_{n \neq n_{0}}\left(g_{n}\right)_{\mathcal{R}} .
$$

Then there exists a sequence of functions $\left\{\varphi_{n}\right\}_{n=1}^{\infty} \subset \mathcal{R}$ such that $h=\varphi_{n_{0}} \cdot g_{n_{0}}$ and $h=\Sigma_{n \neq n_{0}} \varphi_{n} \cdot g_{n}$. It is easy to see that $h \cdot \varphi_{n_{0}} \cdot g_{n_{0}}=$ const $_{0}$, which means that $h=$ const $_{0}$.

\section{References}

[1] F. Azarpanah, Essential ideals in $C(X)$, Period. Math. Hungar., 31(2) (1995), 105-112. 
[2] J. Brown, Almost continuous Darboux functions and Reed's pointwise convergence criteria, Fund. Math., 86 (1974),1-7.

[3] A. M. Bruckner, Differentiation of real functions, Springer - Verlag (1978).

[4] A. M. Bruckner, J. G. Ceder, Darboux continuity, Jbr. Deutsch. Math. Verein, 67 (1965), 93-117.

[5] D. Doliwa and R. J. Pawlak, On the almost continuity of the diagonal of functions, Real Analysis Exch., 23.2 (1997-98), 459-470.

[6] R. Engelking, General topology, Polish Scientific Publishers (PWNWarszawa) (1977).

[7] L. Gillman and M. Jerison, Rings of continuous functions, Springer-Verlag (1976).

[8] O. A. S. Karamzadeh and M. Rostami, On the intrinsic topology and some related ideals of $C(X)$, Proc. Amer. Math. Soc., 93(1) (1985), 179-184.

[9] K. R. Kellum, Almost continuous functions on $I^{n}$, Fund. Math., 79 (1973), 213-215.

[10] K. R. Kellum and B. D. Garret, Almost continuous real functions, Proc. Amer. Math. Soc., 33 (1972), 181-184.

[11] J. Lipiński, On Darboux points, Bull. Acad. Pol. Sci. Ser. Math. Astronom. Phys.. 26, No 11 (1978), 869-873.

[12] T. Natkaniec, Almost continuity, habilitation thesis, Bydgoszcz (1992), $1-131$.

[13] H. Pawlak and R. J. Pawlak, Fundamental rings for classes of Darboux functions, Real Analysis Exch., 14 (1988-89), 189-202.

[14] R. J. Pawlak, Przeksztatcenia Darboux, habilitation thesis, Acta Univ. Łódź, (1985).

[15] R. J. Pawlak, On rings of Darboux functions, Coll. Math., 53 (1987), 289-300.

[16] J. Stallings, Fixed point theorem for connectivity maps, Fund. Math., 47 (1959), 249-263. 
[17] A. Tomaszewska, On the set of functions possessing the property (top) in the space of Darboux and Światkowski functions, Real Analysis Exch., 19.2 (1993-94), 465-470.

[18] R. Viertl, Über die Struktur von Ringen messbarer Funktionen mit algemeinen Ringen als Bildbereich, Ann. Univ. Sci. Budapest Eotvos Sect. Math., 24 (1981), 45-49.

[19] J. Young, A theorem in the theory of functions of a real variable, Rend. Circ. Math. Palermo, 24 (1907), 187-192.

[20] L. Zajíček, Sets of $\sigma$-porosity and sets of $\sigma$-porosity(q), Časopis Pěst. Mat., 101 (1976), 350-359.

[21] L. Zajíček, Porosity and $\sigma$-porosity, Real Analysis Exch., 13 (1987-88), $314-350$. 